\title{
Tratamiento de la Esquizofrenia, de la Psicosis Maniaco-Depresiva y del Alcoholismo con Clorpromacina
}

\author{
Marino Querol L. y Miguel A. Chicata (")
}

\section{INTRODUCCIÓN}

El emplso de todo tratamiento implica una actitud filosófica sobre la causa de la enfermedad que se pretende curar. Los tratamientos psiquiátricos no escapan a esta regla. En efecto, El nihilismo terapéutico corresponde a la época en que el enfermo mental era considerado un poseído o ur alienado. A mediados y fines del siglo pasado se sentaron los fundamentos del organismo en psiquiatría $\mathrm{y}$, en consecuencia, se emplearon los métodos de tratamiento más diversos y concordantes con la supuesta causa orgánica de la enfermedad; en el atán de encontrar el remedio eficaz para los irastornos mentales, los investigadores han ensayado infinidad de substancias, drogas, hormonas, técnicas quirúrcjicas y los más variados métodos terapéuticos, con los que se ha pretendido conseguir efectos curativos. El organicismo trajo consigo la reacción contraria y, desde principios de este siglo, se dejó sentir la influencia psicogenetista con desmedro de aquel. En la actualidad, en la mente del psiquiatra coexisten ambos criterios; sin embargo, debemos reconocer que los tratamientos más efectivos de las psicosis son de base organicista y los aportes que día a día se hacen en este terreno, tienen también $=s a$ tendencia.

Uno de los pasos más importantes en Psiưuictría íié el descubrimiento de los sedantes del sistema nervioso; su aplicación hizo que los establecimientos psiquiátricos dejaran de tener el aspecto carcelario, tan tristemente célebre, y pudieran convertirse

(*) Jeles de Clínica de la Cáledra de Psiquialría, Caledrálico Principal Titular, Prol. Honorio Delgado. 
en hospitales para enfermos menlales. La clorpromacina es una adquisición más en este sentido. Debe hacerse resaltar que con su uso se ha desvirtuado un hecho desfavorable de observación diaria, como ser, el que todo tratamiento psiquiátrico tenía algo de doloroso e inhumano, algo de punitivo para el enfermo; esta vez los propósitos humanitarios del clínico se han puesto al servicio del enfermo mental y se ha consequido, no sólo la sedación sino la remisión de diversos cuadros psiquiátricos sin que el paciente sufra con el tratamiento; por otra parte, el uso de la clorpromacina viene permitiendo que el hospital para enfermos mentales se convierta en un establecimiento de readaptación humana.

\section{MATERIAL Y METODOS}

En este trabajo se expone los resultados del tratamiento con clorpromacina en 50 pacientes esquizofrénicos, 12 con psicosis maníaco-depresiva y 9 alcohólicos crónicos con psicosis sintomática. Todos los enfermos son varones y han recibido tratamiento completo en el pabellón 18 del Hospital "Víctor Larco Herrera". (")

Hemos utilizado la clorpromacina de la casa Specia, conocida en el mercado ccn el nombre de Largactil. El medicamento ha sido administrado por vía oral, salvo en el caso de enfermos muy agitados en quienes sa utilizó, durante los primeros días, la vía intramuscular.

El esquema general del tratamiento en todos los casos ha sido el siguiente: se comienza con dosis mínimas, variables según el cuadro a tratar; luego se aumenta progresivamente hasta alcanzar la dosis máxima en el tiempo de una a cuatro semanas; esta dosis es mantenida hasta obtener los mayores electos, después de lo cual se disminuye paulatinamente hasta llegar a la dosis mínima que permita mantener los beneficios logrados; todo este período lo denominamos de tratamiento activo y la media aritmética diaria de la dosis total de clorpromacina administrada durante el mismo, la denominamos dosis media. El tismpo durante el cual se administra la dosis mínima lo distinguimos como perícdo de sostenimiento.

En un comienzo el uso de la clorpromacina estaba restringi do sólo a aquellos casos en los que habian fracasado tratamien

- Servicio Pensionado de Varones, Médico Jele Prol. H. Delgado, a quien los AA. agradecen por su orientación y eslírulc invalorables. 
tos clásicos, pese a que ellos fueron lo suficientemente intensus y prolongados. Posteriormente los buenos resultados obtenidos hi. cieron que utilizáramos la droga como tratamiento de comienzo, sea sola o asociada a los tratamientos de choque.

En los 50 esquizofrénicos hay 31 paranoides, 5 calatónicos, 4 hebefrénicos, 3 simples, 5 paranoide-catatónicos, un hebefrenocutatónico y un hebefreno paranoide. Las edades varían ontre 15 y 59 años, el tiempo de enfirmedad entre 1 mes y 16 años. Counc puede verse en el cuadro 1, 13 tenian menos de un ciñc de enfermedad y 37 , más de uno. El $54 \%$ de los de menos de 1 año y $89 \%$ de los más de uno, habian sido previomente traiados cori unc o más de los siguientes tratamientos: insulina, electroshoque, leucolomía, bombeo del líquido cefalc-raquídeo y piro-hepátics. El período de tratamiento activo ha durado entre 24 y 261 días. Las dosis diarias de clorpromacina han variado: las medias entre 100 y 475 mgs., las máximas entre 100 y 700 mgs. La dosis de sostenimiento ha sido generalmente de $100 \mathrm{mgs}$. al día. La clorprona. cina ha sido empleada como tratamiento único en 26 esquizistrénicos, asociada al Electrochoque en 13 y a la insulina o a ésta más electrochoque en 11. La clorpromacina sola, que luera utilizada por primera vez por Delay y col., fué administrada a los: pacientes que no habian respondido favorablemente a los trata. mientos clásicos ya mencionados y a aquellos en quienes los tratamientos de choque estaban contraindicados (fractura de la tibia derecha en un caso, tuberculosis pulmonar activa en otro). La La asociación de clcrpromacina al electrochoque se ha hecho en los casos con sintomas catatónicos y paranoides de gran intensidad. Al principio ulilizamos la clorpromacina para morigerar la excitación psicomotriz del precoma y del despertar del coma insulínico, como propugna $\mathrm{H}$. Holf. En vista de los buenos resultados abtenidos y teniendo en mente el planteamiento que se expondrá después, se aumentó la dosis diaria de clorpromacina hasta lograr que el paciente se mantuviera tranquilo y silencioso en el curso de la cura con insulina; al mismo tiempo, fué necesario reducir la dosis de esta última, para evitar comas prolongados. A este tipo de tralamiento fueron sometidos aquellos pacientes que no respondieron favorablemente a la clorpromacina sola o asociada al electrochoque y a las formas paranoides de peor pronóstico. En los casos en que ha sido necesario se ha dado Fenergan y barbitúricos con el objeto de favorecer el sueño, sobre todo du- 
rante los primeros días y cuando la dosis máxima de clorpromacinä era Empleada durante largo tiempo. Los polvos de datura strámonium y al Fenergan han sido utilizados para aminorar e incluso prevenir el parkisonismo farmacológico que se ha presentado en gran número de pacientes. El Fミnergan también ha sido útil para yugular las manifestaciones dérmicas de tipo alérgico que, felizmente, en ningún caso obligaron a suspender el tratamiento. En 9 casos da agitación extrema se administró reserpina, entre 1 y 4 mgs. diarios, durante un período promedio de un mes. En todos los casos hemos administrado vitaminas, especialmente del complejo B. y laxantes suaves, a base de bilis de buey, para combatir el estreñimiento que se presenta durante los tratamientos prolongados y dosis altas.

Las edades de los pacientes con psicosis maníaco-depresiva varíon entre 17 y 74 años, dミ ellos, de los 12 maníacos depresiwos, 7 estaban en fase maníaca y 5 eran melancólicos. Cinco maníacos y un melancólico pres ミntaron fases anteriores. El tiempo comprendido entre el comienzo de la fase y el del tratamiento con clorpromacina, varía entre 15 días y 4 meses en los maníacos y de 3 a 8 meses en los melancólicos. El período de tratamiento activo fué de 12 a 68 días, en los casos de manía, y de 48 a 126 días en las melancolías; las dosis medias en las manías han variado entre 8 y 450 mgs. diarios, las máximas entre 100 y 500 mgs.; en las melancolías, las dosis medias han sido de 25 a 220 mgs. diarios y las máximas de 25 a 300 mgs.

$\mathrm{D}$ e los 7 maníacos, en dos se utilizo la clorpromacina sola, en 4 se le asoció al electrochoque en uno de jstos últirincs y en el $7^{\circ}$

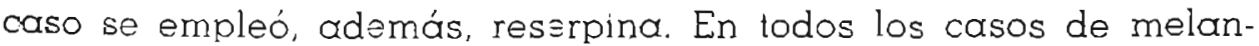
colía se aplicó el electrochoque. El Fenergan y los barbitúricos se administraron como medicación sintomática.

En los alcohólicos crónicos, la edad varía entre 27 y 57 años. El tiempo de duración de la psicosis sintomática hasta el comienzo del tratamiento, ha variado entre 15 dias y 4 meses. El período activo de la cura ha sido de 10 a 45 días, no habiéndose utilizado en ningún caso dosis de sostenimiento. Las dosis diarias han sido: la media de 25 a 150 mgs. Y la máxima de 50 a 200 mgs. En todos los casos se ha empleado vitaminas, especialmente del complejo B y lipotrópicos. En tres casos fué necesario hacer aplicaciones de electrochoque. Para el insomnio se utilizó barbitúricos y Fenergan. 
RESULTADOS

Los resultados oblenidos con el tratamiento se clasifican como: remisión completa ( $R C$ ), cuando el estado mental del paciente $=s$ idéntico al de antes de enfermar; remisión social (RS), si la condición psíquica del enfermo permite la convivencia familiar y la realización de labores productivas de nivel más o menos semejante al de las que realizaba antes de la enfermedad; mejoría (M), cuondo se han abtenido modificaciones del estado mental que favcrecen una mejor convivencia hospitalaria, pero no permiten la readaptación familiar ni extra-hospitalaria de cualquier naturaleza; sin mejoría (SM), aquellos casos en los que no se ha conseguido una modificación ostensible en el cuadro psicótico, asi como, aquelios en quienes los cambios oblenidos no constituyeron aigo favorable. Debemos hacer resaltar que no equiparamos los conceptos de remisión completa y curación; la discusión que ellos suscita sobrepasa los límites de este trabajo.

En el grupo de esquizórénicos, como puede apreciarse en el cuadro No. 1 , hay $85 \%$ de remision $=$ s completas y $15 \%$ de remisiones sociales entre los pacientes de menos de un año de enfermedad. Entre los da más de un año de enfermedad, hay $24 \%$ do rerrisiones completas, $32 \%$ de remisiones sorialos, $35 \%$ de mejorías, $3 \%$ que no mejoraron y $6 \%$ de fallecidos; cifras estas algo superiores a las obtenidas por Labhart. En relación con la forma clínica, los pacientes paranoides, catatónicos y catatónico-paranoides, respondieron mejor al tratamiento en comparación con lcs hebefrénicos, simples y con las formas hebefrénicas mixtas. Los tralamientos de choque combinados con clorpromacina, según nuestra casuística, son más electivos que las curas con clorpromacina sola; ellos pued:n deberse, a que esta droga ha sido empieada sola en un mayor porcentaje de pacientes en quienes fracasaron previamente otros tratamientos, o bien, como se planteará en la discusión, a que la clorpromacina y los tratamientos de choque potencian sus efectos.

La acción de la clorpromacina se manifiesta en primer lugar sobre los trastornos de las tendencias instintivas y de la vida afectiva. Entre los efectos sobre las primeras, es primordial su acción tranquilizadora o ataráxica; mejorando inmediatamente el contacto interpersonal (Baruk y col.) la agitación desaparece paulatinamente en el término de una semana, siempre que las dosis 
empleadas sean lo sulicientemente altas para s: $\equiv \mathrm{r}$ efectivas. En la esfera afectiva disminuye la excitabilidad, se normaliza la espontaneidad así como el cambio de los afectos; el efecto de la clorpromacina sobre la alectividad ha hecho que Sarró la denomine drcga ortotímica; determinados estados afectivos en particular, tales como el miedo y la angustia disminuyen de intensidad e incluso desaparecen en algunos casos, en los que todavía no hay modificaciones favorables de las anormalidades de otras funciones psíquicas, tales como el pミnsamiento y la percepción. En consecuencia el enfermo se relacionará mejcr con el ambiente, su sociabilidad aumenta y la psicoterapia se ve fuvoreciala. Por lo general concomitantemente con los cambios de sentimiento, se apre. cia una mengua y luego desaparición de los trastornos de la percepción, en el curso de la 4ta. o 6ta. semana de tratamiento, lo que es muy claro para las pseudopercepciones auditivas y visuales, no siéndolo tanto para las olíatorias, de la senbilidad general e interna, que persisten en relación con los trastornos d $l \equiv 1$ pensamiento y de la conciencia del yo, las cuales, si bien se modifícan €n el primer mes de cura, sólo dssaparecen algunas semanas más tarde. Cabe anotar que los trastornos del censamiento y muy especialmente las delusiones, persisten sin modificarse en las primeras semonas y en esta forma coexisten con una mejoría en las tendencias instintivas, en el sentimiento y en la percepción. Po'steriormente, la delusión amengua en intensidad, pero, $p \cong r s i s t e$ en el sujeto la convicción plena acerca de su $r \approx a l i d a d$, si bien atirma haber mejorado por el hecho de existir un debilitamiento de lo que clínicamente se conoce como delusiones. En ura fase más avanzada del tratamiento el sujeto reconoce ia anormalidad de sus delusiones, acerca de cuya verosimilitud su convicción no es inquebrantable, ya que son modificables con la psicoterapia. En un estadio ulterior, en los casos de remisión completa y en algunos de remisión social, el sujeto trata $d$ s sus delusiones como de un acontecimiento pasado y anormal, sustentando las razones de su sinrazón. En otros casos de remisión social y de mejoría, las que fueran delusiones se presentan deformadas, sin el carácter dミlusorio original, dado que el paciente las considera anormales y en cierta forma inverosímiles; de tal manera que cuando se presentan no les da importancia, las reconoce de absurdas y adopta una actitud de indiferencia onte ellas. Es necesario recordar que el enfermo tiene amnesia de las delusiones que apa- 
recen en las crisis de agitación, en cambio recuerda las que persistieron después de dichas crisis.

Si se enfoca los efectos de la clorpromacira considerando su acción sobre el sistema nervioso central y el neurovecietativo, sミobserva que desde los primeros días produce cambios somáticos de intensidad variable, según la dósis utilizada. Estos cambios se traducen principalment: por una lentitud en la motilidad general, posteriormente animia, cparición de movimientes involuniarios, síntomas estriados y otros de tipo parasimpático, lo que coniorma un cuadro de parkinsonismo farmacológico, presente en mayor o menor grado, en $\equiv 164 \%$ de los esquizofrénicos de nuestra serie; de acuerdo con Decourt, Flügel y Lehmann, consideramos que no se trata de una compliccción sino de un efecto secundario de la droga. Además, debemos m:ncionar las siguientes com. plicaciones que se presentaron, avanzado el tratamiento active: en 7 casos, figmentación melánica en la piel de la cora (en dos de ellos simultáneamente aparecieron pigmentaciones en la mucosa yugal); en 4 , entorpecimiento de la conciencia, en oiros 4 , vómitos y diarreas, en dos neumopatía aguda; en otros dos, brctes febriles no atribuibles a procesos infecciosos, en uno atrofias musculares y en otro edema localizado cuando tomaba simultáneamente reserpina, lo que concuerda con las observaciones que a Este respecto ha hecho Farley del grupo de Overholsser. No se ha presentado ningún caso de ictericia. En algunos se presentó moderada leucopemia con ligera eosinofilia. Estos cambios físicos van acompañados de las modificaciones psíquicas ya descritas, las cuales scn tanto más favorables, cuanto más intensos y prolongados han sido los trastornos orgánicos. DEbemos considerar que la clorpromacina a dosis elevadas produce una agresión tóxlca, a la que el organismo reacciona con profundos cambios somáticcs, humorales y psíquicos, sierdo las células del sistema nervioso y, en especial, las de la substancia reticular las más sensi. bles a este tipo de agresiones, dada su mayor actividad. Los cam. bios producidos en el organismo por la clorpromacina son a ve. ces tan intensos, que ponen $\triangleq n$ peligro la vida del paciente. Is vuelta a dosis pequeñas o la suspensión del tratamiento en Estos casos, deja un saldo favorable que se aprecia en una mejoría o en una remisión de la psicosis, con desmedro da su estado físico. como hacen notar Laborit y col. En otros casos los cambios orgá. nicos son irreversibles y el suj:to \{allece; tal como ccurrió con un 
esquizofrénico de nuestra casuística, que estaba en el cuarto mes de tratamiento con 50 mgs. de clorpromacina y 4 mgs. de reserpina diarios. En este paciente el parkinsonismo farmacológico era muy marcado, su estado lísico era regular, de pronto presentó filebre alta (40\%), shock y colapso cardíaco, falleciendo dos días después de suprimides las àrocias del tratamiento. Otro paciente de 26 años, falleció al fin de la segunda semana de tratamiento, curando recibía 150 mgs. diarios de clorpromacina asociada a essopo. lamina; el diagnóstico clínico fué bronconeumonía y anasarca. Consideramos que sólo el primer deceso descrito puede atribuírse a la clorpromacina.

En la psicosis maníacondepresiva los resultades del tratamien. to con clorpromacina son espectaculares en las fuses maníacas. En éfecto, de los 7 casos tratalos, 5 remitieron complelamente; 2 fallecieron, uno o conisecuencia da una insuficiencia cardiaca descompensada, con hipertensión arterial; el otro, que padecía de bronquiectomía falleció con síntomas bronconeumónicos pocxs horas después de la aplicación d:l electrochoque; ninguna de ambas muertes farecen ser directamentes causadas por ia clorpromacina. Los cambios de la sintomatología psíquica que se producen con el tratamiento de la clorpromacina en la manía, son tenómenos lógicamente similares a los descritos para la esquizotrenia, con. la diferencia fundamental de que, la modificación del cuadro y la desaparición de los síntomas, son mucho más rápidos en la manía, especialmente en lo que atañe a la modificación tavorable de los trastornas del pensamiento, ya que, en esta enfermedad son an su mayor parte sintomas secundarios. Con el tratamiento, los trastomos desaparecieron entre los 10 y 30 días, su-

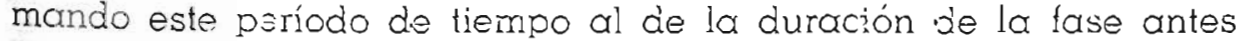
de ser tratada, se aprecia que, en los casos de remisión completa Y que habian presentado lases anteriores, la tratada con clorpromacina fué la de menor duración. En la melancolía, de 5 casos tratados, los 5 remitieron completamente en un perícdo que varió entre 30 y 60 días. En dos de ellos se usó ia clorpromacina como hipnógeno y en los restantes simplemente por sus efectos ataráxiCos, dada la angustia de los pacientes. Su uso fué, pues, con criterio sintomático y consideramos que los buenos resultados obtenidos sobre los síntomas depresivos se deben al electrochoque, que sigue siendo el tratamiento de elección en estos casos. 
Con el mismo criterio de medicación sintomática se aàminis. tró la clcrpromacina a los 9 alcohólicos, cuyas psicosis y los demás trastornos inherentes a la intoxicación alcohólica desaparecieron por compleio. Los efectos de la droga no escapan a los lineamientos fenomenológicos descritos antericrmente, ya que los síntomas que primero se modifican son la agitación, la angustia, la inquietud, el insomnio, la inapetencia, sigue luego la acción sobre los trastornos de la percepción y del persamiento. Es notable también el ef:cto sobre los temblores y finalmente, una vez desaparecidos los sintomas de la psicosis aguda, la droga favorece la buena disposición del alcohólico para permanecer en el hospital, sin incurrir en los atentados contra la disciplina que con tanta frecuencia ocasionan estos pacientes.

En el curso de la cura con clorpromacina y cuando la dosis administrada era de sostenimiento, 7 esquizofrénicos y un melancólico sufrieron una recaída, mejorando el cuadro tan pronto se aumentó la dosis del medicamento. En un caso de manía recrudeció la acitación cuando, en los primeros días de tratamiento, se suspendió bruscamente la administración de la clorpromacina.

El control ulterior de los 38 esquizofiénicos que egresaron del hospital sólo ha sido posible veríficarlo en 18 casos; 10 paranoides, un simple, dos catatónicos, un hebefreno catatónico y un he$b \Leftarrow f r e n o$ paranoide. De los 18 casos controlados 16 siguen en remisión entre dos meses y un año de haber concluído su tratamiento activo; siete de éstos siguen tomando la dosis de sostenimiento bajo vigilancia médica; sclamente un esquizofrénico simple y un paranoide han recaído, después de un mes y un año, respectivamente, de haber terminado el tratamiento activo.

DISCUSION

$\mathrm{D}$ э lo que antecede, de la experiencia de todos nosotros y del acúmulo de publicaciones sobre la clorpromacina, puede docirse que, nos encontramos frente a un producto de acción muy singular y efectiva sobre la esquizofrenia, la psicosis maníacodepresiva y sobre las psicosis alcohólicas. Hemos visto como la clorpromacina produce, en cada uno de los tres grupos nosolć-

- Dicho control ofrece grandes dificultades que han sido salvadas gracias a la efecliva colaboración prestada por las Asistentas Sociales, Srlas. Teresa Blanco, Beatriz Cuentas e Hilda Gómez. 
gicos mencionados, electos que desda el punto de vista fenomenológico son similares. Electivamente, la droga tiene una acción inhibidora sobre los sintomas de excilación en general y sobra trastornos secundarios de! pensamiento. Los electos del medicamento ofrecen algunas dilerencias; asi, en la esquizofrenia, la clorpro. macina a dosis elevadas actía sobre sintomas primarios tales como las delusiones, los trastornos del censamiento esquizofrénicos, el anormal distanciamiento interpersonal tan característico da estos entermos y que muy a menudo es básico para el diagnésíico.

Con relación al liempo de enlermedad en la esquizofrenics, les mejores resultados se observon en los casos de menos de un año, lo que está de acuerdo con el pianteamiento pronóstico de $\mathrm{U}$. Brounmühl tratándose de la insulinoterapia. El período de tratamiento activo en la esquizefrenia debe ser prolongado, de 2 a 4 meses, en cambio el de sostenimiento, consideramos que debe ser mantenido indefinidamente con el fin de evitar las recaílas. La clorfromacina seria caro el esquizofrénico, lo que la digilal es para el cardíaco crónico, la insulina para el diabético o is $\mathrm{m}$ sdiación anticonvulsiva para el epiléptico. Los resultados poco sa. tistactorios obtenidos en el Perú por Trelles y Saavedra A,. Seguín y Castro, y Sal y Rosas, Jeri y Sánchez. Garcia, a! ulilizar la clorpromacina en el tratamisnto de la esquizolrenia probablemien. te se deban a que las dosis empleadas fusron pequeñas y demasiado corla la duración del tralamiento. Posteriomente, Querol y col. cbluvieron mejores resultados empleando dosis más altas y tratamientos de mayor duración.

Los efectos dei tratamiento en la manía también se inanifies. tan co: dnsie liavadas ( 500 mas.) en cambic el período de trotamiento activo ha sido siemcre de corta duración. En los casis de mania grave risistentes a los tratamientos con clorpromacina - electrouthcque, ia ascciación de ambos da mejores resultados. lo que concuerda con la observación de Carrier y col.

Las reccidas se han presentado al bajar las josis o al inie. rrumpir el tratamiento en tcrma prematura. Consideramos que la duracion total de la cura, debe ser equivalente a la duración de la tase husta : I mcinento de empezar el tratamiento. Creemos que, en la melancolía, la ciorpromacina actúa sólo como un coadyuvante en el tratamionio de determinados sintomas, siendo el electrochoque el principal agente ds la remisión, de alli que las dosiz empleadas son pequeñas. 
Sobre el modo y punto de acción de la clorpromacina, se han planteado diferentes argumertos con base experimental y clínica. La mayoría de los investigadores, tales como P. Decourt, Delgado, Lear y colaboradores, Lehmann y otros, consideran que el punto de acción principal $\approx s$ la substancia reticular del tronco encefálico y ello se debería, gracias a la abundancia celular y asc ciativa de esa zona. Ei nivel de acción de la clorpromacina iría ascendiendo proporcionalmente a la dósis. $D €$ este modo se explicaría cómo a pequeñas dosis inhibe al sistema reticulci: culiva dor del tronco encefálico y prculuce desaparición de los temblores en los alcohólicos, en cambio, a dósis mayores, al actuar sobre los ganglios basales produce movimientos involuntarios. Con ello también se explicaría que la acción de esta droga se manifriesta pri. mero sobre el nivel de vigilancia favoreciendo el sueño, y que luego, a medida que su acción se deja sentir en nivelss más altos, en algunos casos, ese eflecto es menor e incluso se invierte. Así por ejemplo, algunos de nuestros pacientes que recibían do. sis altas de clorpromacina y barbitúricos con las que dormían saiisfactoriamente en los primeros días, después de algún tiempo se quejaban de insomnio pertinaz, a pesar de seguir recibiendo las mismas dosis o aun mayores; como se ve, el efecto era inverso. Un enfermo de nuestra casuística (esquizofrenia paranoide) que sólo fué tratado con Largactil y Serpasol, presentó insomnio rebelde $a$ diversos barbitúricos (Fenobarbital, Amytal), en el curso de la segunda semana de tratamiento, pocos días después el insomnio era acompañado de gran intranquilidad motora y de excitación psíquica. Según P. Decourt, en estos cásos raros, "el tono de su actividad encefálica tiene (por una razón que ignoramos), una inversión del equilibrio habitual entre los sistemas facilitadores e inhibidores", acción debida a la influencia narcobiótica. Los efectos inversos anotados se han producido, con más frecuencia, en los casos en que se ha asociado la reserpina a la clorpromacina, pero no constituyen la regla en el tratamiento con clorpromacina, sola o asociada a los tratamientos de chcque, durante el cual sólo excepcionalmente hemos observado en forma clara las 3 fases que describen Flügel, Gade y Heinrich y otros. Como hemos anotado anteriormente, las dosis elevadas de clorpromacina producen una agresión tóxica -más marcada si se asocia la clorpromacina a la reserpina o a otras drogas- que se manifiesta en el organismo por profundos cambios físicos, en espe- 
cial en el sistema nervioso central, en el reurovegetativo, principalmente en el parosimpático, el que a su vez origina hiperfunción secretoria de diversas glándulas. Todos estos mecanismos puestos en juego están en relación con lo qua Selye describe como efectos del "Stress" y con los llamados fenómenos de Reilly. La mayor parte de estos cambios desaparecen cuando se suspende la administración de la droga, pero, en algunos cascs, hacen peligrar la vida del enfermo o condicionan la producción de lesiones definitivas (temblor persistente en la porción distal de los miembros superiores y atrofias musculares en las manos en uno de nuestros casos). Al éecto tóxico de la asociación de la clorpromacina con la reserpina puede atribuírse sólo la muerte de uno de los esquizofrénicos. La mortalidad por efectos tóxicos en el grupo de esquizolrénicos tratados con clorpromacina sería pués del $2 \%$, cifra bastante aceptable y que es comparable con la de mortalidad por tratamiento de chooue.

En los cuadros 1 y 2 puede verse que los resultados del tratamiento con clorpromacina en las esquizofrénicos, son mejores que los de otros tratamientas sir clorpromacina (Querol). En el cuadro 2 podemos apreciar que, en dos cirupos de esquizofrénicos similares, en cuanto al porcentaje de formas clínicas, en cada uno de ellos los resultados porcentuales del tratamiento con clorpromacina son superiores a los obtenidos sin clorpromacina; pese a que en este grupo el porcentaje de pacientes de menos de un año de tiempo de enfermedad es mucho menor. En este mismo cuadro podemos apreciar que en grupos similares de pacientes -por lo que alañe a formas clínicas y tiempo de enf三rmedad-. con clorpromacina y electrcchoque se obtiene mejores resultados que con clorpromacina sola, lo que ya había sido observado por Gade y Heinrich y Bergouignan y col.; pero los resultados óptimos se obtienen en el grupo tratado con clorpromacina e insulina - con clorpromacina y tratamiento mixto -...pese a que en este grupo se incluyeron pacientes con pronóstico dasíavorable-. De lo dicho se desprende la hipótesis de que, los efectos de la clorpromacina y los tratamientos de choque, especialmente los de insulina, se potencian - tomando este término en el sentido en que queda expuesto en la ley de Burgi- para aquellos síntomas mentales sobre los cuales tienen efecio favorable ambas substancias (Trumart y Boehm). Un dato más que sustenta esta hipótesis, es la disminución de las dosis de insulince, necesarias para 
TRATADOS COMPARATIVOS OBTENIDOS EN DOS GRUPOS DE ESQUIZOFRENICOS TRATADOS, UNO Y OTRO SIN CLORPLOMACINA. DE ACUERDO CON EL TIEMPO DE ENFERMEDAD

\begin{tabular}{|c|c|c|c|c|c|c|c|c|c|c|c|c|c|c|}
\hline \multirow[t]{2}{*}{ METODOS } & \multicolumn{7}{|c|}{ MENOS DE UN AÑO } & \multicolumn{7}{|c|}{ MAS DE UN AÑNO } \\
\hline & $\begin{array}{c}N^{\prime} \\
\text { Pac. }\end{array}$ & $\begin{array}{l}\text { Con } \\
\text { Tra. } \\
\text { Pre }\end{array}$ & $\begin{array}{l}\text { RC } \\
\%\end{array}$ & $\begin{array}{l}\mathrm{RS} \\
\%\end{array}$ & $\begin{array}{l}M \\
\%\end{array}$ & $\begin{array}{l}\mathrm{SM} \\
\%\end{array}$ & $\mathrm{Fa}$ & $\begin{array}{c}N^{!} \\
\text {Pac. }\end{array}$ & $\begin{array}{l}\text { Con } \\
\text { Tra. } \\
\text { Pre }\end{array}$ & $\begin{array}{l}\mathrm{RC} \\
\%\end{array}$ & $\begin{array}{l}\text { RS } \\
\% \%\end{array}$ & $\begin{array}{l}M \\
\%\end{array}$ & $\begin{array}{c}S M \\
\%\end{array}$ & $\mathrm{Fa}$ \\
\hline CON CLORPROMACINA & 13 & 7 & 85 & 15 & - & - & - & 37 & 33 & 24 & 32 & 35 & 3 & 6 \\
\hline $\begin{array}{l}\text { OTROS TRATAMIENTOS } \\
\text { SIN CLORPROMACINA }\end{array}$ & 46 & - & 35 & 26 & 17 & 22 & - & 57 & -- & 5 & 23 & 16 & 53 & B \\
\hline
\end{tabular}

RESULTADOS GLOBALES OBTENIDOS CON DIFERENTES TIPOS DE TRATAMIENTO EN DOS GRUPOS DE ESQUIZOFRENICOS SIMILARES

En cuanto al porcentaje de formas clínicas en cada uno de ellos.

CUADRO No. 2

\begin{tabular}{|c|c|c|c|c|c|c|c|c|}
\hline \multirow{2}{*}{$\begin{array}{l}N^{\prime} \text { de } \\
\text { Pacient. }\end{array}$} & \multirow{2}{*}{$\begin{array}{r}\text { Tiemp. } \\
-1^{4}\end{array}$} & \multirow{2}{*}{$\begin{array}{l}\text { Ent. } \\
+\quad 1^{1}\end{array}$} & \multirow[t]{2}{*}{ TIPO TRATAMIENTO } & \multicolumn{5}{|c|}{ RESULTADOS \% } \\
\hline & & & & $\mathrm{RC}$ & $\mathrm{RS}$ & M & SM & Fa. \\
\hline 103 & $45 \%$ & $35 \%$ & sin closprom. & 18 & 24 & 17 & 39 & 2 \\
\hline 50 & $28 \%$ & $74 \%$ & con clorprom. & 40 & 28 & 26 & 2 & 4 \\
\hline 26 & $27 \%$ & $73 \%$ & clorpr. sola & 34 & 31 & 31 & - & 4 \\
\hline 13 & $20 \%$ & $80 \%$ & clorpr. + $\mathrm{BE}$ & 46 & 15 & 23 & 8 & 8 \\
\hline 11 & $27 \%$ & $73 \%$ & clorpr. + I o Mkt. & 46 & 36 & 18 & - & - \\
\hline
\end{tabular}

producir el coma en aquellos facientes en quienes se empszó el iratamiento con esta substancia y luego se asoció la clorpromacina; en aquellos pacientes a quienes no se disminuye la dosis de insulina, cuando se asocia la clorpromacina, hay tendencia al despertar lento y dilícil, y propensión al ccma prolongado; esto ocurrió en dos de nuestros enfermos.

Podemos, pues, terminar diciendo que si bien $\triangleq 1$ tratamiento con clorpromacina no es causal en sentido estricto, constituye uno de los mejores medicamentos de que dispone el psiquiatra actual. mente y dabemos añadir que la asociación de la clorpromacina 
a la insulina y electrochoque da resultados terapéuticos hasta ahora no logrados con ningún otro método. La enfermedad mental ya no es un problema tan sombrío desde la aparición y el uso adecuado de los llamados narcobióticos.

\section{CONCLUSIONES}

1.-.Se ha utilizado la clorpromacina sola o asociada con tratamientos de choquə, en 50 esquizctrénicos, 7 maniacos, 5 melancólicos y en 9 alcohóiicos con psicosis sintomática.

2.- Los resultados obtenijos han sido: en los esquizoírénicos, $40 \%$ de remisiones completas, $28 \%$ de remisiones sociales, $26 \%$ de mejorías, $2 \%$ sin mejoría y $4 \%$ de fallecidos. En la manía, to. dos los pacientes remitieron en pocos días y la duración total de la fase tratada fué menor que las anteriores. En la melancolía todos los pacientes remitieron por efecto del electrcchoque, y la clorpromacina sólo fué un tratamiento coadyuvant:. Todos los casos de psicosis alcohólicas remitieron, siendo la droga particularmente útil en los cuadros parancides y otros síntomas de excitación en las tendencias instintivas y de la vida afectiva.

3.-Se compara los resultados del tratamiento de la esquizofrenia con clorpromacina y los obtenidos con otras curas clásicas, apreciándose resultados favorables con el primer método en el $98 \%$ de les casos; en cambio, con los otros tratamientos ( $\sin$ clorpromacina) sólo se beneficia el $71 \%$.

4.-Los mejores resultados del tratamiento con clorpromacina en la esquizolrenia, han sido obtenidos mediante la asociación de esta droga con la insulina o con el tratami=nto mixto (insulina más electrochoqus). Dado que, con la asociación de clorpromacina con electrochoque también se obtienen mejores resultados que con la clorpromacina sola, se plantea la hicótesis de que los efectos de los tratamientos de choque y los de la cjorpromacina se potencializan.

5.--De los 71 pacientes tratados, 2 esquizofrénicos y dos maníaccs fallecieron; de ellos los dos maníacos y un esquizotrénico murieron por complicaciones varias debidas al estado previo del sujeto. Sólo la muerte de un esquizofrénico puede ser atribuida a la clorpromacina asociado a la reserpina; la mortalidad por es. to tratamiento en la esquizotrenia sería pues dei $2 \%$.

6.-.-Se discute el modo y punto de acción de la clorpromacina. 


\section{REFERENCIAS BIBLIOGRAFICAS}

Braunmuir, A V.- "Insulinshock und Heilkrampt in der Psychialrie". Wissenschafliche Verlagsgesellschatt, Siuttgari, 1947.

Baruk, H., LauNay, I. ei Berges, J.: "La thèrapeutique par la chlorpromazine Fhysiolos:o experimentaic. Physiologie clinique. Résullats therapeutiques él progrés de la thérapeutique csychiatrique". Ann. Med.- Psychol., 113: 705. 772, 1955.

bergouinan, M., D'Aulnay, j. el Malineau, R.: Relouchs á nolre experience du Largactil en Psychiaire. Jour. Med. Bordeaux, No. 3, 254-261, 1955.

Carrier J. Guyotat, J, el Grandmottet, J.: Electro-choc el Largactil dans les accés maniaques. journ. Med. Lyon, No. 238, 950-951, 1954.

Decovpt, Ph.: Acciones larmacodinámicas y fisiológicas asociadas a los narcobiólicos. Rev. Psiquiat. Psicol. Med. 2: 3. 233-241, 1955.

Decount, Ph.: Ensayos de estudios leóricos sobre cierlos problemas planteados por la aplicación de la terapéutica narcobiótica en psiquiatría. Rev. Psiquiat. Psicol. Med. 2: 3, 290-310. 1955.

Delay, J., Deniker, y Harl, J. M.: "Utilisation en thérapéutioue d'une phenothazine d'aciion centiale éleclive (4560 RP)". Ann. Med. Psychol., 110: 112. $113,1952$.

Delay, J., Deniker, P. el Harl, J. M.: "Trailement des élats d'excitation el d" hibernothérapie". Ann. Med.- Psychol., 110: 267-273. 1952

DELGADO HoNozio: "The trealmenl of compulsive neurosis with chlorpromazine". Centennial Papers, Saint Elizabeths Hospilal, Washinglon, 1956.

Delgaco, Honorio: "Sur nolre expérience avec la chlorpromazine". Colloque in. ternational sur la chlororomazine el medicamenls reuroleptiques en ihérapeutique psychialrique. Paris 20, 21 ei 22 oclobre 1955. La Presse Med., 64: 13-14. 1956.

Flugel, F.: "Ucher medikamentor erzengie parkinsonahuliche zustandcilder". Med. Klinik, 15: 694.635, 1955.

Flugel, F.: "Erlahrungen mit Megaphen in der psychiatrischen und neurologischen klinik". Verhandiungen der Deutschen Gesellchall Fürfinnere Medizin, 1954.

Gade, E. B. Y Heinrich, K.: "Klinische Beobachlungen bei Megaphenbehandlung in der Psychialrie", Nervenarzl. No. 2, 1955.

Hoff, Hans: Die thercpie der schizophrenia. Wiener Klinishe Wchst. CC. J., 20. $345.352,1954$.

LABHARDT F.: "Die Largacliltherapie bei Schizcphrenien und andere Psychotischen Zusiânden". Sehvreilae: Arch. Neur. Psych. 73: 1/2 338-345, 1954.

LABHARDT, F.: Technik, Nebenerscheinungen un Komplikalionen der Largaciil. therapic. Schwelzer. Arch. Psych. 73: 1/2 338-345, 1954.

Laborit H. y Huguenáa P.: "Práclica de la hibernolerapia en Medicina y cirugía, Editorial Pupul, Barcelona, 1955.

Lear, E. Chiron, A. and Pallin, I.: Some inleresting pharmacodynamic proper. ties of chlorpromazine. J. Clin. Exp. Psychop. 17: 147-152, 1956.

Lemmañ, H. E.: Neurophysiologic Activity of chlorpromazine in clinical Use. J. Clin Exp. Psychop. 17: 129-141. 1956. 
Overholser, W., Waldrop F., Riesenmax, R., fong T., farley O. QHiorrman J.: Chlorpromazine and Reserpine, symposium on expariences at Saint Elizabelhs Hospilal Medical Annais ef the District of Columbia. No. 25, No. 5, mayo, 1956. Querol., M.: "El tralamien:o de la esquizolrenia", Revisia de Neuro-osiquiatría, Lima, $N^{2} 1955$.

Querol, M., Samanez F. y Almeida, M.: "La clorpromacina en el tratamiento do la esquizolrenia y la fsicosis maniaco-depresiva", (en prensa).

SARro, R.: "Técnica, complicaciones y resullados de la lerapéutica con la cior. promacina. Rev. Psiquial. Psicol. Med. 2: 3, 215.222, 1955.

SAL Y Rosas, F. Jert. R. y Sásciszz García: "Primesos resullados del iratamienio de Largaclil en los eniermos mentales". Rev. Neuio. Psiq. 17: 358, 1954.

SegufN, C. A. y Castro, R.: Experiencia en el empleo de la clorpromacina. Rev. Neuro-Psiq. 17: 357, 1954.

Trummert, W. y Boelim, G.: Problemas terminológicos concernientes a los nar. cobióticos. Rev. Psiquial. Psicol. Meà., 2: 3, 223-232, 1955.

Trelles, 3. O. y SaAvedra, A.: "Cura de sueño con Largaclij en el tratamiento de algunos desordenes mentales". Rev. Neuro.Psiq. 17: 125, 1954. 